\title{
Perturbation to Symmetry and Adiabatic Invariants of General Discrete Holonomic Dynamical Systems
}

\author{
P. WANG* AND H.-J. ZHU ${ }^{\dagger}$ \\ College of Physics and Electronic Engineering \\ Xinjiang Normal University, Urumqi, Xinjiang 830054, China
}

(Received June 24, 2010)

\begin{abstract}
This paper investigates perturbation to the Noether symmetry of discrete holonomic nonconservative dynamical systems on a uniform lattice. Firstly, we give the Noether theorem of system. Secondly, both criterion of perturbation to the Noether symmetry and the Noether adiabatic invariants of system are obtained. Finally, an example is given to illustrate these results.
\end{abstract}

PACS: $02.20 . \mathrm{Sv}, 11.30 .-\mathrm{j}, 45.05 .+\mathrm{x}$

\section{Introduction}

Symmetries play important roles in mathematics, physics and mechanics. Since Noether unveiled the profound relations between symmetries and conservation laws, many researches on them were done [1-15]. Recently, symmetry theories have been extended to discrete mechanics and equations [16-25].

As we know, even tiny changes in symmetry, named as perturbation to symmetry, are of great importance for physical systems. Based on the definition of adiabatic invariants, the relationship of perturbation to symmetry with adiabatic invariants are constructed. It offers an opportunity for the quasi-integrability in dynamical systems $[26,27]$. Therefore, perturbation to symmetry and adiabatic invariants became a popular subject recently. The notion of approximate conservation laws was introduced with regards to approximate Noether symmetry by Baikov et al. [28]; Kara et al. [29, 30] extended Baikov's ideas. Fu and Chen et al. [31, 32] studied the perturbation to the Lie symmetry and adiabatic invariants. Zhang et al. [33] deduced a new type of adiabatic invariants from perturbation to the Lie symmetry in 2006. Luo [34] gave another new type of adiabatic invariants called the Lutzky adiabatic invariants lately. These studies further inspire interests in research about adiabatic invariants [35-37].

However, researches about perturbation to symmetry and adiabatic invariants are all considered in continuous

\footnotetext{
* e-mail: wpxy04@yahoo.com.cn

$\dagger$ e-mail: hjzhu@xjnu.edu.cn
}

systems. In this paper, we firstly study the Noether symmetry and Noether exact invariants of discrete nonconservative dynamical systems. Secondly, we study perturbation to symmetry and adiabatic invariants of discrete dynamical systems. Finally, we give an example to illustrate the application of these results.

\section{Definitions and notations}

We consider [19] the space $Z$ of sequence $\left(t, q^{s}, \dot{q}^{s}\right)$, and in the space $Z$, we define a map (differentiation), obeying to the rule: $D(t)=1, D\left(q^{s}\right)=\dot{q}^{s}, D\left(\dot{q}^{s}\right)=\ddot{q}^{s} . D$ is the action of the first order linear differential operator

$$
D=\frac{\partial}{\partial t}+\dot{q}^{s} \frac{\partial}{\partial q^{s}}+\ddot{q}^{s} \frac{\partial}{\partial \dot{q}^{s}}+\ldots \quad(s=1, \ldots, n) .
$$

An arbitrary value of parameter $h>0$ is fixed and with the help of the tangent field (1), we form the operators of discrete translation to the right and left

$$
\underset{+h}{S}=\mathrm{e}^{h D} \equiv \sum_{i=0}^{\infty} \frac{h^{i}}{i !} D^{i}, \underset{-h}{S}=\mathrm{e}^{-h D} \equiv \sum_{i=0}^{\infty} \frac{-h^{i}}{i !} D^{i}
$$

The operators $\underset{+h}{S}, \underset{-h}{S}$ commute with each other, while $S \cdot S=S \cdot S=1$.

Using the simplest invariant lattice $h$ in $t$-direction, we introduce a pair of linear difference operators $S_{+h}$ and $S_{-h}$, which are defined by

$$
\underset{+h}{S} q^{s}=q^{s+}, \quad \underset{-h}{S} q^{s}=q^{s-} .
$$

Moreover, with the help of $S_{+h}$ and $S_{-h}$, we can form a pair of operators of discrete (finite-difference) differentiation to the right and left

$$
\underset{+h}{D}=\frac{S_{+h}-1}{h}=\sum_{i=1}^{\infty} \frac{h^{(i-1)}}{i !} D^{i},
$$




$$
\underset{-h}{D}=\frac{1-S_{-h}}{h}=\sum_{i=1}^{\infty} \frac{(-h)^{(i-1)}}{i !} D^{i} .
$$

The operators $S_{+h}, S_{-h}, D_{+h}$ and $D_{-h}$ commute in any combination, while $D_{+h}=D_{-h} S_{+h}, D_{-h}=D_{+h} S_{-h}$, and it follows corresponding finite-difference Leibniz rule:

$$
\begin{aligned}
& \underset{+h}{D}(F G)=\underset{+h}{D}(F) G+F \underset{+h}{D}(G)+h \underset{+h}{D}(F) \underset{+h}{D}(G), \\
& \underset{-h}{D}(F G)=\underset{-h}{D}(F) G+F \underset{-h}{D}(G)-h \underset{-h}{D}(F) \underset{-h}{D}(G) .
\end{aligned}
$$

\section{Noether symmetry and exact invariants of discrete holonomic nonconservative dynamical systems}

Suppose that the configuration of a mechanical system is determined by $n$ generalized coordinates $q^{s}$ ( $s=$ $1, \ldots, n)$, the Lagrangian of the system is $L(t, \boldsymbol{q}, \dot{\boldsymbol{q}})$. For a positive integer $k$, the discrete Hamilton action of the system is the map defined by:

$$
M_{d}=\sum L_{d}\left(t, q^{s}, q_{k}^{s}\right) h,
$$

where $L_{d}\left(t, q^{s}, q_{k}^{s}\right)$ is the corresponding discrete Lagrangian and the corresponding difference derivative to right and left are

$$
\begin{aligned}
& q_{k}^{s}=\underset{+h}{D}\left(q^{s}\right)=\frac{q^{s+}-q^{s}}{t^{+}-t}=\frac{q^{s+}-q^{s}}{h}, \\
& q_{k}^{\frac{s}{}}=\underset{-h}{D}\left(q^{s}\right)=\frac{q^{s}-q^{s-}}{t-t^{-}}=\frac{q^{s}-q^{s-}}{h} .
\end{aligned}
$$

The infinitesimal transformations are introduced with respect to time and generalized coordinates as

$$
t^{*}=t+\Delta t, \quad q^{s *}\left(t^{*}\right)=q^{s}(t)+\Delta q^{s}
$$

$$
(s=1, \ldots, n) \text {. }
$$

Expanding above formulation, we have

$$
\begin{aligned}
& t^{*}=t+\epsilon \xi_{00}^{\alpha}\left(t, q^{s}, \dot{q}^{s}\right), \quad q^{s *}=q^{s}+\epsilon \xi_{s 0}^{\alpha}\left(t, q^{s}, \dot{q}^{s}\right), \\
& \quad(s=1, \ldots, n ; \alpha=1, \ldots, \gamma),
\end{aligned}
$$

where $\epsilon$ is infinitesimal parameter and $\xi_{00}^{\alpha}\left(t, q^{s}, \dot{q}^{s}\right)$, $\xi_{s 0}^{\alpha}\left(t, q^{s}, \dot{q}^{s}\right)$ constitute the Lie group of infinitesimal transformation. The infinitesimal transformation operator (i.e. the generalized Noether-type operator) is introduced as

$$
\begin{aligned}
& \left.X=\xi_{00}^{\alpha} \frac{\partial}{\partial t}+\xi_{s 0}^{\alpha} \frac{\partial}{\partial q^{s}}+\underset{+h}{D}\left(\xi_{s 0}^{\alpha}\right)-q_{k}^{s} \underset{+h}{D}\left(\xi_{00}^{\alpha}\right)\right] \frac{\partial}{\partial q_{k}^{s}} \\
& +\ldots+h \underset{+h}{D}\left(\xi_{00}^{\alpha}\right) \frac{\partial}{\partial h} .
\end{aligned}
$$

We used the simplest invariant lattice which is regular with the constant step $h$.

Under the infinitesimal transformation (8), the discrete Hamilton action becomes

$$
M_{d}^{*}=\sum L_{d}\left(t^{*}, q^{s *}, q_{k}^{s *}\right) h^{*},
$$

where $h^{*}=\left(1+D_{+h}(\Delta t)\right) h=\left(1+\epsilon D_{+h}\left(\xi_{00}^{\alpha}\right)\right) h$ and $\Delta$ expresses the total variation.

Definition 1. If Hamilton action of discrete holonomic nonconservative system is generalized quasi-invariant under the infinitesimal transformation (8), i.e.

$$
\begin{aligned}
& \Delta M_{d}=\sum L_{d} h-\sum L_{d}^{*} h^{*} \\
& =-\sum_{s=1}^{n} \underset{+h}{\left.\underset{D}{D}\left(\Delta G_{d}\right)+Q_{d}^{s} \delta_{d} q^{s}\right\} h}
\end{aligned}
$$

we call (8) the discrete analogue of generalized Noether quasi-symmetry transformation. $G_{d}=G_{d}\left(t, q^{s}, q_{k}^{s}\right), Q_{d}^{s}=Q_{d}^{s}\left(t, q^{s}, q_{k}^{s}\right)$ are the discrete gauge function and discrete nonconservative forces. $Q_{d}^{s} \delta_{d} q^{s}$ are the discrete analogue of the virtual work for nonconservative generalized forces.

Making use of the relation $\Delta q^{s}=\delta_{d} q^{s}+q_{k}^{s} \Delta t, \Delta q_{k}^{s}=$ $\delta_{d} q_{k}^{s}+\left(q_{k k}^{\frac{s}{k}}\right) \Delta t$ (where $\left.q_{k k}^{\frac{s}{k}}=D_{-h} D_{+h} q^{s}\right)$ and the commute relation $D_{+h}\left(\delta_{d} q^{s}\right)=\left(\delta_{d} q_{k}^{s}\right)$, and after direct calculations by applying the Leibniz rule of (forward) difference as the discrete derivative, we can obtain the following formulation form (12):

$$
\begin{aligned}
& \frac{\partial L_{d}}{\partial t} \Delta t+\frac{\partial L_{d}}{\partial q^{s}} \Delta q^{s}+\frac{\partial L_{d}}{\partial q_{k}^{s}}\left(\underset{+h}{D}\left(\Delta q^{s}\right)-q_{k}^{s} \underset{+h}{D}(\Delta t)\right) \\
& \quad+L_{d} \underset{+h}{D}(\Delta t)+Q_{d}^{s} \delta_{d} q^{s}+\underset{+h}{D}\left(\Delta G_{d}\right)=0 .
\end{aligned}
$$

Substituting the infinitesimal transformation (9) into Eq. (13), and considering the independence of parameter $\epsilon$ of the Lie group, we have

$$
\begin{aligned}
& \frac{\partial L_{d}}{\partial t} \xi_{00}^{\alpha}+\frac{\partial L_{d}}{\partial q^{s}} \xi_{s 0}^{\alpha}+\frac{\partial L_{d}}{\partial q_{k}^{s}}\left[\underset{+h}{D}\left(\xi_{s 0}^{\alpha}\right)-q_{k}^{s} \underset{+h}{D}\left(\xi_{00}^{\alpha}\right)\right] \\
& \quad+L_{d} \underset{+h}{D}\left(\xi_{00}^{\alpha}\right)+Q_{d}^{s}\left(\xi_{s 0}^{\alpha}-q_{k}^{s} \xi_{00}^{\alpha}\right)+\underset{+h}{\underset{D}{D}}\left(G_{d}^{\alpha}\right)=0,
\end{aligned}
$$

where we made use of $\Delta G_{d}=\sum_{\alpha=1}^{\gamma} \epsilon_{\alpha} G_{d}^{\alpha}$. From definition 1 , we have:

Criterion 1. If the infinitesimal transformation (8) satisfies (13), it is called the Noether generalized quasi-symmetry transformation of the discrete holonomic nonconservative dynamical systems.

Criterion 2. If the infinitesimal transformation (9) satisfies (14), it is called the Noether generalized quasi-symmetry transformation of the discrete holonomic nonconservative dynamical systems.

We name (14) the discrete analogue of generalized Noether-type identity for this discrete holonomic nonconservative system. Equation (14) can be expressed as

$$
\begin{aligned}
& \frac{\partial L_{d}}{\partial t} \xi_{00}^{\alpha}+\frac{\partial L_{d}}{\partial q^{s}} \xi_{s 0}^{\alpha}+\frac{\partial L_{d}}{\partial q_{k}^{s}}\left[\underset{+h}{D}\left(\xi_{s 0}^{\alpha}\right)-q_{k}^{s} \underset{+h}{D}\left(\xi_{00}^{\alpha}\right)\right] \\
& +L_{d} \underset{+h}{D}\left(\xi_{00}^{\alpha}\right)+Q_{d}^{s}\left(\xi_{s 0}^{\alpha}-q_{k}^{s} \xi_{00}^{\alpha}\right)+\underset{+h}{\underset{D}{D}\left(G_{d}^{\alpha}\right)} \\
& =\xi_{00}^{\alpha}\left[\frac{\partial L_{d}}{\partial t}+\underset{-h}{D}\left(q_{k}^{s} \frac{\partial L_{d}}{\partial q_{k}^{s}}-L_{d}\right)-q_{k}^{s} Q_{d}^{s}\right] \\
& +\xi_{s 0}^{\alpha}\left[\frac{\partial L_{d}}{\partial q^{s}}-\underset{-h}{D}\left(\frac{\partial L_{d}}{\partial q_{k}^{s}}\right)+Q_{d}^{s}\right]+\underset{+h}{D}\left\{\xi_{00}^{\alpha} \underset{-h}{S}\left(L_{d}\right)\right.
\end{aligned}
$$




$$
\left.+\left[\xi_{s 0}^{\alpha}-\underset{-h}{S}\left(q_{k}^{s}\right) \xi_{00}^{\alpha}\right]_{-h}^{S}\left(\frac{\partial L_{d}}{\partial q_{k}^{s}}\right)+G_{d}^{\alpha}\right\}=0 .
$$

If there exists generalized quasi-extremal equation for discrete holonomic nonconservative system, such as

$$
\begin{gathered}
\xi_{00}^{\alpha}\left[\frac{\partial L_{d}}{\partial t}+\underset{-h}{D}\left(q_{k}^{s} \frac{\partial L_{d}}{\partial q_{k}^{s}}-L_{d}\right)-q_{k}^{s} Q_{d}^{s}\right] \\
+\xi_{s 0}^{\alpha}\left[\frac{\partial L_{d}}{\partial q^{s}}-\underset{-h}{D}\left(\frac{\partial L_{d}}{\partial q_{k}^{s}}\right)+Q_{d}^{s}\right]=0 .
\end{gathered}
$$

We can obtain the difference analogues of generalized Euler-Lagrange equations

$$
\underset{-h}{D}\left(\frac{\partial L_{d}}{\partial q_{k}^{s}}\right)-\frac{\partial L_{d}}{\partial q^{s}}=Q_{d}^{s}
$$

and energy equations

$$
\frac{\partial L_{d}}{\partial t}+\underset{-h}{D}\left(q_{k}^{s} \frac{\partial L_{d}}{\partial q_{k}^{s}}-L_{d}\right)-q_{k}^{s} Q_{d}^{s}=0 .
$$

Correspondingly, the discrete analogue of conservation law of the system is

$$
\begin{aligned}
& \underset{+h}{D}\left[\xi_{00}^{\alpha} \underset{-h}{S}\left(L_{d}\right)+\left[\xi_{s 0}^{\alpha}-\underset{-h}{S}\left(q_{k}^{s}\right) \xi_{00}^{\alpha}\right]\right. \\
& \left.\quad \times \underset{-h}{S}\left(\frac{\partial L_{d}}{\partial q_{k}^{s}}\right)+G_{d}^{\alpha}\right]=0,
\end{aligned}
$$

namely Noether exact invariants

$$
\begin{aligned}
I_{d}^{0} & =\xi_{00}^{\alpha} \underset{-h}{S}\left(L_{d}\right)+\left[\xi_{s 0}^{\alpha}-\left(q_{k}^{s}\right) \xi_{00}^{\alpha}\right] \\
& \times \underset{-h}{S}\left(\frac{\partial L_{d}}{\partial q_{k}^{s}}\right)+G_{d}^{\alpha}=\text { const. }
\end{aligned}
$$

The difference Eqs. (19) and (20) are called the difference analogue of the Noether conservation laws associated with such a discrete holonomic nonconservative system. The difference Eqs. (19) and (20) form the invariant schemes on regular lattice $h$ and thus coincide with the difference Noether conservation laws.

We should point out that the first item of (16) "disappears" in continuous limit since the operator in brackets tends to zero as $h \rightarrow 0$.

Theorem 1. If the Lie group (9) of the infinitesimal transformations of the discrete system (16) on a uniform mesh $h$, are the Noether generalized quasi-symmetry transformation, in the condition that the discrete gauge functions $G_{d}^{\alpha}$ exist, then the holonomic nonconservative system has the discrete analogue of the Noether conserved quantity (19) or (20).

Theorem 2. If the Lie group (9) of the infinitesimal transformations of the discrete system (17) and (18) on a uniform mesh $h$, are the Noether generalized quasi-symmetry transformation, in the condition that the discrete gauge functions $G_{d}^{\alpha}$ exist, then the holonomic nonconservative system has the discrete analogue of the Noether conserved quantity (19) or (20).

We call theorems 1 and 2 the discrete analogue of generalized Noether theorems of discrete holonomic nonconservative systems.

\section{Perturbation to symmetry and adiabatic invariants of discrete holonomic nonconservative dynamical systems}

Suppose systems (17) and (18) are perturbed by small quantity $\epsilon W_{d}^{s}=\epsilon W_{d}^{s}\left(t, q^{s}, q_{d}^{s}\right)$, the equations of discrete holonomic nonconservative dynamical systems become

$$
\underset{-h}{D}\left(\frac{\partial L_{d}}{\partial q_{k}^{s}}\right)-\frac{\partial L_{d}}{\partial q^{s}}=Q_{d}^{s}+\epsilon W_{d}^{s}
$$

and

$$
\frac{\partial L_{d}}{\partial t}+\underset{-h}{D}\left(q_{k}^{s} \frac{\partial L_{d}}{\partial q_{k}^{s}}-L_{d}\right)-q_{k}^{s}\left(Q_{d}^{s}+\epsilon W_{d}^{s}\right)=0
$$

Due to the action of $\epsilon W_{d}^{s}$, the primary symmetries and invariants of systems (17) and (18) may vary. The variation is assumed as a small perturbation based on the symmetrical transformation of the initial system, then $\xi_{0}^{\alpha}, \xi_{s}^{\alpha}$ which denote the new generators after being perturbed, can be expressed as

$$
\begin{aligned}
& \xi_{0}^{\alpha}=\xi_{00}^{\alpha}+\epsilon \xi_{01}^{\alpha}+\epsilon^{2} \xi_{02}^{\alpha}+\ldots, \\
& \xi_{s}^{\alpha}=\xi_{s 0}^{\alpha}+\epsilon \xi_{s 1}^{\alpha}+\epsilon^{2} \xi_{s 2}^{\alpha}+\ldots
\end{aligned}
$$

The new generators satisfy

$$
\begin{aligned}
& \frac{\partial L_{d}}{\partial t} \xi_{0}^{\alpha}+\frac{\partial L_{d}}{\partial q^{s}} \xi_{s}^{\alpha}+\frac{\partial L_{d}}{\partial q_{k}^{s}}\left[\underset{+h}{D}\left(\xi_{s}^{\alpha}\right)-q_{k}^{s} \underset{+h}{D}\left(\xi_{0}^{\alpha}\right)\right] \\
& \quad+\underset{+h}{L_{d}}\left(\xi_{0}^{\alpha}\right)+Q_{d}^{s}\left(\xi_{s}^{\alpha}-q_{k}^{s} \xi_{0}^{\alpha}\right)+\epsilon W_{d}^{s}\left(\xi_{s}^{\alpha}-q_{k}^{s} \xi_{0}^{\alpha}\right) \\
& \quad+\underset{+h}{D}\left(G_{d}^{\alpha}\right)=0 .
\end{aligned}
$$

If we assume

$$
G_{d}^{\alpha}=G_{d 0}^{\alpha}+\epsilon G_{d}^{\alpha}+\epsilon^{2} G_{d 2}^{\alpha}+\ldots,
$$

and substitute (23) and (25) into (24), we have

$$
\begin{gathered}
\frac{\partial L_{d}}{\partial t} \xi_{0 m}^{\alpha}+\frac{\partial L_{d}}{\partial q^{s}} \xi_{s m}^{\alpha}+\frac{\partial L_{d}}{\partial q_{k}^{s}}\left[\underset{+h}{D}\left(\xi_{s m}^{\alpha}\right)-q_{k}^{s} \underset{+h}{D}\left(\xi_{0 m}^{\alpha}\right)\right] \\
+\underset{+h}{L_{d}}\left(\xi_{0 m}^{\alpha}\right)+Q_{d}^{s}\left(\xi_{s m}^{\alpha}-q_{k}^{s} \xi_{0 m}^{\alpha}\right)+W_{d}^{s}\left(\xi_{s m-1}^{\alpha}\right. \\
\left.-q_{k}^{s} \xi_{0 m-1}^{\alpha}\right)+\underset{+h}{D}\left(G_{d m}^{\alpha}\right)=0 \quad(m=0,1,2, \ldots) .
\end{gathered}
$$

When $m=0$, the condition $W_{d}^{s}=0$.

The generalized Noether-type operator for perturbed system becomes

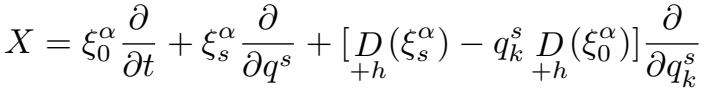

$$
\begin{aligned}
& +\ldots+h \underset{+h}{D}\left(\xi_{0}^{\alpha}\right) \frac{\partial}{\partial h} .
\end{aligned}
$$

Substituting (23) into (27), we have

$$
X=\epsilon^{m} X_{m},
$$

where

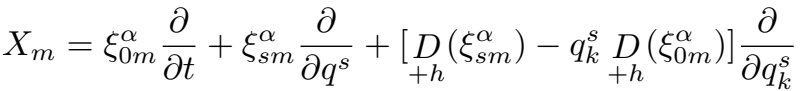

$$
\begin{aligned}
& +\ldots+h \underset{+h}{D}\left(\xi_{0 m}^{\alpha}\right) \frac{\partial}{\partial h} .
\end{aligned}
$$

So we can give the criterion of perturbation to the Noether symmetry of the system 
Criterion 3. For perturbed discrete systems (21) and (22), if the infinitesimal transformation generators $\xi_{0 m}^{\alpha}, \xi_{s m}^{\alpha}$ satisfy (26), and there exists gauge function $G_{d m}^{\alpha}=G_{d m}^{\alpha}\left(t, q^{s}, q_{k}^{s}\right)$, the corresponding variety of the Noether symmetry of discrete holonomic nonconservative dynamical system is called perturbation to the Noether symmetry.

According to the definition of adiabatic invariants in Ref. [29], we can give

Definition 2. For systems (21) and (22), if a physical quantity $I_{d}^{z}\left(t, q^{s}, q_{d}^{s}, \epsilon\right)$ satisfies

$$
\underset{+h}{D}\left(I_{z}\right)=\mathrm{O}\left(\epsilon^{z+1}\right)
$$

where

$$
I_{d}^{z}=I_{d 0}^{0}+\epsilon I_{d 1}^{1}+\ldots+\epsilon^{z} I_{d z}^{z},
$$

$I_{d}^{z}$ is called a $z$-th-order adiabatic invariant of systems.

Based on the definition 2 and the criterion 3, we have the following theorem:

Theorem 3. For the systems (21) and (22), which are perturbed by a small physical quantity $\epsilon W_{d}^{s}$, if the generators $\xi_{0 m}^{\alpha}, \xi_{s m}^{\alpha}$ of the infinitesimal transformations are perturbation to the Noether symmetry (i.e. the generators $\xi_{0 m}^{\alpha}, \xi_{s m}^{\alpha}$ satisfy criterion 2), the systems have discrete analogue of $z$-th-order adiabatic invariants which can be written in the following form:

$$
\begin{aligned}
I_{d}^{z} & =\sum_{m=0}^{z} \epsilon^{m}\left\{\xi_{0 m}^{\alpha} \underset{-h}{S}\left(L_{d}\right)+\left[\xi_{s m}^{\alpha}-\left(q_{k}^{s}\right) \xi_{0 m}^{\alpha}\right]\right. \\
& \left.\times \underset{-h}{S}\left(\frac{\partial L_{d}}{\partial q_{k}^{s}}\right)+G_{d m}^{\alpha}\right\} .
\end{aligned}
$$

When $z=0, W_{d}^{s}=0$ holds.

Proof: Calculating the discrete derivative of $I_{d}^{z}$, taking consideration of (26) and following the Leibniz rule, we have:

$$
\begin{aligned}
& \underset{+h}{D}\left(I_{d}^{z}\right)=\xi_{0 m}^{\alpha} \underset{-h}{D}\left(L_{d}\right)+\left(\xi_{s m}^{\alpha}-q_{k}^{s} \xi_{0 m}^{\alpha}\right) \underset{-h}{D}\left(\frac{\partial L_{d}}{\partial q_{k}^{s}}\right) \\
& \quad+\underset{-h}{D}\left(q_{k}^{s}\right) \xi_{0 m}^{\alpha} \frac{\partial L_{d}}{\partial q_{k}^{s}}-\xi_{0 m}^{\alpha} \frac{\partial L_{d}}{\partial t}-\xi_{s m}^{\alpha} \frac{\partial L_{d}}{\partial q^{s}} \\
& \quad-Q_{d}^{s}\left(\xi_{s m}^{\alpha}-q_{k}^{s} \xi_{0 m}^{\alpha}\right)-W_{d}^{s}\left(\xi_{s m-1}^{\alpha}-q_{k}^{s} \xi_{0 m-1}^{\alpha}\right) \\
& \quad=\xi_{0 m}^{\alpha}\left[{ }_{-h}^{D}\left(L_{d}-q_{k}^{s} \frac{\partial L_{d}}{\partial q_{k}^{s}}\right)-\frac{\partial L_{d}}{\partial t}+q_{k}^{s} Q_{d}^{s}\right] \\
& \quad+\xi_{s m}^{\alpha}\left[\underset{-h}{D}\left(\frac{\partial L_{d}}{\partial q_{k}^{s}}\right)-\frac{\partial L_{d}}{\partial q^{s}}-Q_{d}^{s}\right] \\
& -W_{d}^{s}\left(\xi_{s m-1}^{\alpha}-q_{k}^{s} \xi_{0 m-1}^{\alpha}\right) .
\end{aligned}
$$

Making use of Eqs. (21) and (22), after deduction, we have

$$
\underset{+h}{D}\left(I_{d}^{z}\right)=\sum_{m=0}^{z}\left[\epsilon W_{d}^{s}\left(\xi_{s m}^{\alpha}-q_{k}^{s} \xi_{0 m}^{\alpha}\right)\right.
$$

$$
\left.-W_{d}^{s}\left(\xi_{s m-1}^{\alpha}-q_{k}^{s} \xi_{0 m-1}^{\alpha}\right)\right] .
$$

Expending the above formula and making summation, we obtain

$$
\underset{+h}{D}\left(I_{d}^{z}\right)=\epsilon^{z+1} \sum_{s=1}^{n} W_{d}^{s}\left(\xi_{s z}^{\alpha}-q_{k}^{s} \xi_{0 z}^{\alpha}\right) .
$$

It shows that $D_{+h}\left(I_{d}^{z}\right)$ is in direct proportion to $\epsilon^{z+1}$, thus $I_{d}^{z}$ is discrete analogue of $z$-th-order adiabatic invariants for discrete disturbed holonomic nonconservative systems (21), (22).

\section{Illustrating example}

The dynamical systems with discrete Lagrangian

$$
L=\frac{1}{2}\left[\left(q_{k}^{1}\right)^{2}+\left(q_{k}^{2}\right)^{2}\right]-q^{2}
$$

and nonconservative forces are

$$
Q_{d}^{1}=q_{k}^{1}, \quad Q_{d}^{2}=\frac{t^{+}+t}{2}-q_{k}^{2} .
$$

Let us study its exact invariants and adiabatic invariants.

The discrete analogue of generalized Noether type identity of this system is

$$
\begin{aligned}
& -\xi_{20}^{\alpha}+q_{k}^{1}\left[\underset{+h}{D}\left(\xi_{10}^{\alpha}\right)-q_{k}^{1} \underset{+h}{D}\left(\xi_{00}^{\alpha}\right)\right] \\
& +q_{k}^{2}\left[\underset{+h}{D}\left(\xi_{20}^{\alpha}\right)-q_{k}^{2} \underset{+h}{D}\left(\xi_{00}^{\alpha}\right)\right]+L_{d} \underset{+h}{D}\left(\xi_{00}^{\alpha}\right) \\
& +q_{k}^{1}\left(\xi_{10}^{\alpha}-q_{k}^{1} \xi_{00}^{\alpha}\right)+\left(\frac{t^{+}+t}{2}-q_{k}^{2}\right)\left(\xi_{20}^{\alpha}-q_{k}^{2} \xi_{00}^{\alpha}\right) \\
& +\underset{+h}{D}\left(G_{d}^{\alpha}\right)=0 .
\end{aligned}
$$

It has two groups of solution

$$
\begin{aligned}
& \xi_{00}^{\alpha}=0, \quad \xi_{10}^{\alpha}=\frac{t^{+}+t}{2}, \quad \xi_{20}^{\alpha}=1, \\
& G_{d}^{\alpha}=q^{2}+\frac{t^{+}+t}{2}\left(1-q_{k}^{1}\right)-\frac{1}{2}\left(\frac{t^{+}+t}{2}\right)^{2}, \\
& \xi_{00}^{\alpha}=0, \quad \xi_{10}^{\alpha}=0, \quad \xi_{20}^{\alpha}=1, \\
& G_{d}^{\alpha}=q^{2}+\frac{t^{+}+t}{2}-\frac{1}{2}\left(\frac{t^{+}+t}{2}\right)^{2} .
\end{aligned}
$$

So they are Noether symmetrical. According to (20), we can get discrete Noether conserved laws as

$$
\begin{aligned}
& I_{d 01}^{\alpha}=q^{2}+q_{k}^{2-}+\left(q_{k}^{\frac{1}{k}-} q_{k}^{1}+1\right) \frac{t^{+}+t}{2} \\
& -\frac{1}{2}\left(\frac{t^{+}+t}{2}\right)^{2}=\text { const } \\
& I_{d 02}^{\alpha}=q^{2}+q_{k}^{2}+\frac{t^{+}+t}{2}-\frac{1}{2}\left(\frac{t^{+}+t}{2}\right)^{2}=\text { const . }
\end{aligned}
$$

In the following, we will study the first order adiabatic invariants of system. Suppose the system is perturbed by 
$\epsilon W_{d}^{1}=\epsilon 2 q_{k}^{2} q_{k}^{2}, \quad \epsilon W_{d}^{2}=-\epsilon q^{2}$.

Let $m=1$, then Eqs. (26) give

$$
\begin{aligned}
& -\xi_{21}^{\alpha}+q_{k}^{1}\left[\underset{+h}{D}\left(\xi_{11}^{\alpha}\right)-q_{k}^{1} \underset{+h}{D}\left(\xi_{01}^{\alpha}\right)\right]+\underset{+h}{q_{k}^{2}}\left[\underset{+h}{D}\left(\xi_{21}^{\alpha}\right)\right. \\
& \left.-q_{k}^{2} \underset{+h}{D}\left(\xi_{01}^{\alpha}\right)\right]+L_{d} \underset{+h}{D}\left(\xi_{01}^{\alpha}\right)+q_{k}^{1}\left(\xi_{11}^{\alpha}-q_{k}^{1} \xi_{01}^{\alpha}\right) \\
& +\left(\frac{t^{+}+t}{2}-q_{k}^{2}\right)\left(\xi_{21}^{\alpha}-q_{k}^{2} \xi_{01}^{\alpha}\right)+2 q_{k k}^{2} q_{k}^{2}\left(\xi_{10}^{\alpha}\right. \\
& \left.-q_{k}^{1} \xi_{00}^{\alpha}\right)+q^{2}\left(\xi_{20}^{\alpha}-q_{k}^{2} \xi_{00}^{\alpha}\right)+\underset{+h}{D}\left(G_{d 1}^{\alpha}\right)=0 .
\end{aligned}
$$

We can work out solution as

$$
\begin{aligned}
& \xi_{01}^{\alpha}=1, \quad \xi_{11}^{\alpha}=q^{1}, \quad \xi_{21}^{\alpha}=0, \\
& G_{d 11}^{\alpha}=\frac{1}{2}\left(q^{1}\right)^{2}+q^{2} \frac{t^{+}+t}{2}-\left(q_{k}^{2}\right)^{2} \frac{t^{+}+t}{2},
\end{aligned}
$$

when we make use of the Noether symmetry generators $\xi_{00}^{\alpha}=0, \xi_{10}^{\alpha}=\frac{t^{+}+t}{2}, \xi_{20}^{\alpha}=1$.

We can work out another group of solutions as

$$
\begin{gathered}
\xi_{01}^{\alpha}=1, \quad \xi_{11}^{\alpha}=q^{1}, \quad \xi_{21}^{\alpha}=q_{k}^{2}+\frac{t^{+}+t}{2}, \\
G_{d 12}^{\alpha}=\frac{1}{2}\left[\left(q^{1}\right)^{2}+\left(q_{k}^{2}\right)^{2}\right]-q^{2} \frac{t^{+}+t}{2} \\
+\left(\frac{t^{+}+t}{2}\right)^{3}\left(\frac{1}{3}-\frac{1}{t^{+}+t}\right)
\end{gathered}
$$

when we make use of the Noether symmetry generators $\xi_{00}^{\alpha}=0, \xi_{10}^{\alpha}=0, \xi_{20}^{\alpha}=1$.

The corresponding first order discrete Noether adiabatic invariants are

$$
\begin{gathered}
I_{d 11}^{\alpha}=I_{d 01}^{\alpha}+\epsilon\left\{q^{1} q_{k}^{1}-q^{2}-\frac{1}{2}\left[\left(q_{k}^{\frac{1}{k}}\right)^{2}+\left(q_{k}^{2}\right)^{2}\right.\right. \\
\left.\left.-\left(q^{1}\right)^{2}\right]+\left[q^{2}-\left(q_{k}^{2}\right)^{2}\right] \frac{t^{+}+t}{2}\right\} .
\end{gathered}
$$

and

$$
\begin{aligned}
& I_{d 12}^{\alpha}=I_{d 01}^{\alpha}+\epsilon\left\{q^{1} q_{k}^{1}-q^{2}+q_{k}^{2} q_{k}^{2}-\frac{1}{2}\left[\left(q_{k}^{\frac{1}{k}}\right)^{2}\right.\right. \\
& \left.\left.-\left(q^{1}\right)^{2}\right]+\left(q_{k}^{\frac{2}{2}}\right)^{2}-\left(q_{k}^{2}\right)^{2}\right\}+\left(q_{k}^{2}-q^{2}\right) \frac{t^{+}+t}{2} \\
& \quad+\left(\frac{t^{+}+t}{2}\right)^{3}\left(\frac{1}{3}-\frac{1}{t^{+}+t}\right) .
\end{aligned}
$$

Furthermore, we can obtain higher order adiabatic invariants.

\section{Conclusion}

In this paper, (1) we obtain the Noether exact invariants for discrete holonomic nonconservative systems; (2) we propose both the criterion of the perturbation to the Noether symmetry and the Noether adiabatic invariants of discrete holonomic nonconservative systems. These results can be also extended to discrete nonholonomic dynamical systems.

\section{Acknowledgments}

Project supported by the Foundation for Prior Development Subject of Theory Physics of Xinjiang Normal University and the Research plan of Higher Education Institutions of Xinjiang autonomous region (grant No. XJEDU2010S31).

\section{References}

[1] E. Noether, Kgl. Ges. Wiss. Nachr. Göttingen Math. Phys. 2, 235 (1918).

[2] D.S. Djukić, B.D. Vujanović, Acta Mech. 23, 17 (1975).

[3] W. Sarlet, F. Cantrijn, SIAM Rev. 23, 467 (1981).

[4] L.Y. Bahar, H.G. Kwatny, Int. J. Non-Linear Mech. 22, 125 (1987).

[5] M. Lutzky, J. Phys. A, Math. Gen. 12, 973 (1979).

[6] G.E. Prince, C.J. Eliezer, J. Phys. A, Math. Gen. 14, 587 (1981).

[7] F.X. Mei, Symmetries and Conserved Quantities of Constrained Mechanical Systems, Institute of Technology Press, Beijing 2004 (in Chinese).

[8] S.A. Hojman, J. Phys. A, Math. Gen. 25, L291 (1992).

[9] M. Lutzky, J. Phys. A, Math. Gen. 28, L637 (1995).

[10] J. Goedert, F. Haas, Phys. Lett. A 239, 348 (1998).

[11] Y.X. Guo, S.K. Luo, M. Shang, F.X. Mei, Rep. Math. Phys. 47, 313 (2001).

[12] J.L. Fu, L.Q. Chen, J. Salvador, Y.F. Tang, Phys. Lett. A 358, 5 (2006).

[13] Y. Zhang, Chin. Phys. B 18, 4636 (2009).

[14] J.H. Fang, M.J. Zhang, W.W. Zhang, Phys. Lett. A 374, 1801 (2010).

[15] J.L. Cai, Acta Phys. Pol. A 117, 445 (2010).

[16] S. Maeda, Math. Jpn. 25, 405 (1980).

[17] D. Levi, P. Winternitz, J. Math. Phys. 37, 5551 (1996).

[18] D. Levi, S. Tremblay, P. Winternitz, J. Phys. A, Math. Gen. 34, 9507 (2001).

[19] V.A. Dorodnitsyn, J. Sov. Math. 55, 1490 (1991).

[20] V.A. Dorodnitsyn, R.V. Kozlov, P. Winternitz, J. Math. Phys. 41, 480 (2000).

[21] V.A. Dorodnitsyn, Appl. Numer. Math. 307, 321 (2001).

[22] J.E. Marsden, M. West, Acta Numer. 357 (2001).

[23] P.E. Hydon, Proc. R. Soc. A 456, 2835 (2000).

[24] H.B. Zhang, L.Q. Chen, R.W. Liu, Chin. Phys. 14, $1063(2005)$

[25] J.L. Fu, B.Y. Chen, L.Q. Chen, Phys. Lett. A $\mathbf{3 7 3 ,}$ 409 (2009).

[26] Y.Y. Zhao, F.X. Mei, Symmetries and Invariants of Mechanical Systems, Science Press, Beijing 1999 (in Chinese)

[27] V.A. Baikov, R.K. Gazizov, N.H. Ibragimov, J. Sov. Math. 55, 1450 (1991). 
[28] V.A. Baikov, R.K. Gazizov, N.H. Ibragimov, in: $C R C$ Handbook of Lie Group Analysis of Differential Equations, Vol. 3, Ed. N.H. Ibragimov, CRC Press, Boca Raton, Florida 1996, p. 31.

[29] A.H. Kara, F.M. Mahomed, G. Ünal, Int. J. Theor. Phys. 38, 2389 (1999).

[30] A.G. Johnpillai, A.H. Kara, Int. J. Theor. Phys. 40, 1501 (2001).

[31] J.L. Fu, L.Q. Chen, Phys. Lett. A 324, 95 (2004).

[32] X.W. Chen, Y.M. Li, Y.H. Zhao, Phys. Lett. A 337, 274 (2005).

[33] Y. Zhang, C.X. Fan, F.X. Mei, Acta Phys. Sin. 55, 3237 (2006) (in Chinese).
[34] S.K. Luo, Acta Phys. Sin. 57, 5580 (2007) (in Chinese).

[35] P. Wang, J.H. Fang, X.M. Wang, Chin. Phys. Lett. 26, 034501 (2009).

[36] M.J. Zhang, J.H. Fang, K. Lu, Int. J. Theor. Phys. 49, 427 (2010).

[37] N. Ding, X.F. Chen, J.H. Fang, C.Z. Liu, Phys. Lett. A 373, 3005 (2009). 